\title{
Confección de un modelo predictor para infección de herida operatoria en pacientes adultos intervenidos de artroplastía total de cadera en un hospital de alta complejidad, años 2012 y 2014
}

\author{
Cristian Lara', Marcela Cárcamo', Inés Cerón², Rodrigo Adasme', Cynthia Urquidi' y Gabriel Cavada'
}

\author{
Predictive model preparation for surgical wound infection in adult \\ patients undergoing total hip arthroplasty in high complexity hospital \\ for years 2012 and 2014
}

Introduction: Operative wound infections of patients undergoing total hip arthroplasty have an incidence from $2 \%$ to $5 \%$, generating impact on hospital stay, resource use, prolonged antibiotic therapy, including temporary or definitive sequelae. Objective: To generate a predictive model for surgical wound infection in patients undergoing total hip arthroplasty between 2012 and 2014 at the High Complexity Hospital. Material and Method: Cohort of patients with total hip arthroplasty. A description of the epidemiological variables was made and a predictive model was generated by means of logistic regression. Results: 441 patients were analyzed. The predictive model obtained included the variables: days of post-operative stay (OR 1.11 IC95\% [1.03 - 1.20]), transfusion of at least one unit of red blood cells (OR 3.13 IC95\% [1.17 - 10.86]), diagnosis of previous depression to surgery (OR 5.75 IC95\% [1.32 - 25.32], non-compliance with antibioprophylaxis administration time (OR 5.46 IC95\% [1.68 - 17.78], $\mathrm{P}<0.001$ ) and pseudo $\mathrm{R} 2=0.2293$. Score point of 13 points with sensitivity $44.4 \%$, specificity of $91.6 \%$, LR (+) 5.29, LR (-) 0.61, 1 to 6 points "low risk", 7 to 12 points "medium risk", 13 to 18 points "high risk", from 19 points as "maximum risk". Conclusion: the model presents a good predictive capacity of operative wound infection and adequately represents the cohort under study.

Keywords: Infections; surgical; wound; hip, prosthesis, arthroplasty; model; prediction.

Palabras clave: Infección; herida; prótesis; cadera; artroplastia; modelo; predicción.
'Departamento de Salud Pública y Epidemiología, Universidad de Los Andes. Santiago, Chile. ${ }^{2}$ Departamento de Enfermedades Infecciosas, Escuela de Medicina. Pontificia Universidad Católica de Chile. Santiago, Chile.

Declaración de conflictos de interés reales y potenciales: Los autores declaran que no presentar conflictos de interés reales ni potenciales en la ejecución ni resultados del presente estudio.

Fuente de Financiamiento: Recursos propios del investigador principal.

Recibido: 15 de marzo de 2018 Aceptado: 23 de octubre de 2018

Correspondencia a: Cristian Lara R. cristian.lara.epi@gmail.com.

\section{Introducción}

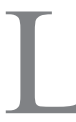

as infecciones asociadas a la atención de salud (IAAS) son frecuentes y costosas ${ }^{1}$. Respecto a las infecciones del sitio quirúrgico (ISQ), son la segunda causa más común de infecciones contraídas por pacientes ingresados en un recinto de atención de salud ${ }^{2}$. Específicamente en las infecciones de la herida operatoria (IHO) de pacientes intervenidos de artroplastía total de cadera (ATC), estas presentan una incidencia desde 2 a $5 \%$; sin embargo, generan un importante impacto en la estadía hospitalaria, uso de recursos, antibioterapia prolongada y secuelas temporales o definitivas en los pacientes que sufren una de estas infecciones ${ }^{3,4}$. En E. U. A., el costo total calculado para este tipo de IHO, proyectado para el año 2020, es de $\$ 1,62$ billones de dólares ${ }^{8}$. Como factores de riesgo descritos se encuentran: la edad avanzada (OR 3,8 [IC95\% 1,1-13,0]), bacteriuria asintomática (OR 3,95 [IC95\% 1,52-10,26]), tiempo quirúrgico prolongado mayor a $4 \mathrm{~h}$ (OR 1,80 [IC95\% $1,25-2,59]$ ), tiempo quirúrgico menor a $60 \mathrm{~min}$ (OR 2,4 [IC95\% 1,4-4,0]) ASA igual o mayor a 3 (OR 2,62
[IC95\% 1,66-4,14]) y post-operatorio en una unidad de cuidado intensivo (OR 6,64 [IC95\%1,24-35,64]) ${ }^{16-18}$. En un estudio multicéntrico de casos y controles se detectó la depresión (HR 1,38 [IC95\% 1,11-1,72]), obesidad (HR 1,73 [IC95\%1,35-2,22]), arritmia cardíaca (HR 1,30 [IC95\%1,11-1,52]) y la presencia de una enfermedad reumatológica (HR 1,71 [IC95\%1,42-2,06]) ${ }^{20}$. Si se analizan estudios en Latinoamérica, específicamente en Brasil, se muestra como factores de riesgo para IHO de ATC: ASA sobre III (OR 2,72 [IC95\%: 1,05-7,02]) y permanencia preoperatoria mayor a 6 días (OR 2,96 [IC95\%: 1,31-6,69] $)^{4}$. Existen varias guías internacionales sobre la prevención de este tipo de infecciones ${ }^{6}$, junto a la descripción de diversos factores de riesgo asociados a IHO; sin embargo, se desconocen estos factores de riesgo en los usuarios de la red de salud pública chilena, tanto nacional como específicamente en la red de atención sur oriente de la Región Metropolitana. El trabajo en materia de prevención está basado en información internacional, que puede no corresponder a la realidad local. Por lo tanto, es fundamental conocer el comportamiento de estos factores por medio de la creación de un modelo 
predictivo de IHO para intervenir y prevenir este problema de una forma concreta, atingente y efectiva. El objetivo general del estudio fue generar un modelo predictivo de IHO en pacientes intervenidos de ATC, entre los años 2012 y 2014.

\section{Material y Método}

Se conformó una cohorte retrospectiva basada en análisis de registros clínicos de pacientes intervenidos de ATC en el Complejo Asistencial Dr. Sótero del Río, entre los años 2012 y 2014. La cohorte incluyó la totalidad de los pacientes intervenidos de ATC o prótesis de cadera (PTC), según el registro estadístico de intervenciones quirúrgicas efectuadas en el período determinado. Se revisaron sus historias clínicas hasta un año posterior a la fecha de la cirugía.

Se excluyeron los pacientes con diagnóstico de patología infecciosa en la articulación previo al procedimiento quirúrgico o que tuvieran algún otro tipo de intervención quirúrgica en el mismo espacio anatómico donde se efectuó la instalación de la prótesis.

Al momento de la revisión de los registros clínicos, se obtuvieron datos respecto al perfil epidemiológico y demográfico de cada uno de los pacientes y factores asociados, junto a la detección de infección de la IHO según criterios del MINSAL vigentes entre los años 2012 y $2014^{12}$. Dentro de las variables extraídas al momento de la revisión de las historias clínicas, se consideraron las siguientes:

Variables basales del paciente: Edad, sexo, peso, talla, presencia de enfermedades crónicas diagnosticadas, consumo de tabaco, uso de corticoesteroides, tratamiento antimicrobiano previo, foco infeccioso concomitante, portación de Staphylococcus aureus, presencia de bacteriuria, cirugía y hospitalización previa hasta un año y motivo de la prótesis.

Variables asociadas a la atención de salud previas a la intervención: Días de hospitalización previos a la cirugía, servicio clínico, registro de cumplimiento de preparación preoperatoria de la piel, hematocrito previo, albuminemia previa, glicemia previa, protrombinemia previa, continencia urinaria.

Variables asociadas a la atención de salud durante el acto quirúrgico: Años de ejercicio de la especialidad del traumatólogo principal, registro de cumplimiento de preparación intraoperatoria de la piel, ASA, tipo de anestesia, tiempo operatorio, tipo de abordaje quirúrgico, lateralidad de la intervención, media de temperatura corporal durante el acto quirúrgico, registro de sangramiento anormal durante la cirugía, tipo, dosis y tiempo transcurrido desde antibioprofilaxis hasta la incisión quirúrgica, cumplimiento de antibioprofilaxis según protocolo local, instalación de drenaje en el lecho quirúrgico, media de glicemia intraoperatoria. Respecto a las variables de tratamiento antimicrobiano (imipenem, amikacina, por ejemplo), se refiere a que hubo pacientes que presentaron una infección concomitante posterior a la intervención quirúrgica y fueron tratados según el esquema de terapia institucional.

Variables relacionadas con atención de salud posteriores a la intervención quirúrgica: Necesidad de transfusión de hemocomponentes, presencia de IHO, días de estadía post-operatorios, servicio clínico de estadía post-intervención, número de curaciones efectuadas en el post-operatorio, hematocrito, glicemia y protrombinemia post-intervención, uso de dispositivos invasivos, continencia urinaria post-intervención, presencia de infección asociada a la atención de salud (IAAS) distinta a IHO, uso y tipo de antimicrobianos administrados en el post-operatorio como tratamiento, destino de egreso, tratamiento odontológico hasta un año posterior a la intervención y estado vital al egreso hospitalario.

\section{Análisis estadístico}

Se efectuó un análisis descriptivo de las variables en estudio, de acuerdo con su naturaleza, utilizando estadísticos de tendencia central, dispersión o proporciones. Posteriormente, se efectuó la determinación de factores asociados a la presencia o ausencia de IHO en pacientes con ATC calculando la medida de asociación odds ratio $(O R)$ por medio de regresión logística univariada, utilizando de forma separada cada una de las variables independientes con IHO. De las variables que se presentaron como factor de riesgo $(\mathrm{OR}>1)$ o protector $(\mathrm{OR}<1)$ con nivel de significación estadística menor o igual a 0,25 (criterio de pesquisa de factores de Hosmer y Lemeshow) ${ }^{24,25}$. Se confeccionó un modelo multivariado saturado, al cual se le agregaron factores de riesgo descritos en la literatura especializada. Para la determinación del modelo predictivo final se comenzó desde un modelo que incluyó todas las variables, en una selección "stepwise", para luego efectuar eliminación progresiva de las variables con un nivel de significación mayor de 0,05 , hasta obtener un modelo compuesto por la menor cantidad de variables explicativas. La capacidad discriminativa del modelo, se evaluó mediante el área bajo la curva ROC. Posteriormente, se efectuó un análisis del modelo predictivo por medio de medidas de resumen del nivel de ajuste incluyendo: logaritmo de verosimilitud del modelo saturado, prueba de significancia de LR, $R^{2}$ de McFadden (crudo y ajustado), Count $R^{2}$ (crudo y ajustado). Finalmente se efectuó un análisis comparativo y de sensibilidad de pérdidas o 
sujetos no considerados en el modelo, para observar si existen diferencias estadísticamente significativas entre los sujetos incluidos o no incluidos en el modelo predictivo final. Para la obtención del "score" de IHO/PTC se utilizó los $O R$ obtenidos de cada una de las variables incorporadas al modelo predictivo final. Para ello, se multiplicó el $O R$ por el valor de su respectiva variable. Posteriormente, se calculó la sensibilidad y especificidad de cada uno de los valores del "score" para determinar un punto de corte que favoreciera la especificidad por sobre la sensibilidad, dado que clínicamente es relevante poder determinar los pacientes que realmente están en riesgo, pudiendo intervenir de forma específica, considerando que el objetivo es prevenir las IHO.

Para la recolección y confección de la base de datos se utilizó programa Microsoft Excel ${ }^{\circledR} 2013$ (Washington, E. U. A.), con licencia comprada por el investigador. Para el análisis estadístico se utilizó el programa STATA ${ }^{\circledR} 13$ (Año 2013, Texas, E.U.A), con licencia otorgada para el investigador por Universidad de Los Andes.

\section{Resultados}

Entre los años 2012 y 2014 se intervino a 500 pacientes de ATC según información obtenida desde el sistema estadístico informático de la tabla quirúrgica. De estos, se excluyeron 55 pacientes por instalación de la prótesis en una zona anatómica con prótesis anterior o con el uso de diferentes técnicas de osteosíntesis, otros 9 pacientes por patología infecciosa de la articulación (artritis séptica) y 10 pacientes intervenidos de otra patología, mal catalogados según codificación de sistema informático de pabellones quirúrgicos. Por lo tanto, se conformó una cohorte de 441 pacientes. Se pudo acceder a los registros clínicos del total de la cohorte durante la recolección de la información.

La mayoría de los pacientes fueron mujeres (68,2\%), en donde más de la mitad de ellas eran hipertensas (57,1\%) y el 15,2\% diabéticas. Respecto a los hombres, representaron $31,7 \%$ de los pacientes, más de la mitad de ellos eran hipertensos (55\%), y 20,7\% diabéticos.

La edad de la cohorte tenía una mediana de 71 años $\left(\mathrm{P}_{25}=63 ; \mathrm{P}_{75}=77 ; \mathrm{RIC}=14\right)$ al igual que para las mujeres, en cambio en los hombres alcanzó los 70 años $\left(\mathrm{P}_{25}=63,5 ; \mathrm{P}_{75}=76 ; \mathrm{RIC}=12,5\right)$. Se observó que el peso en las mujeres fue menor que en los hombres, pero a la vez, tenían menor estatura (Tabla 1).

De los pacientes intervenidos, la mayoría se debió a una artrosis de la articulación $(76,4 \%)$ y por fractura cerrada $(22,2 \%)$. En $72 \%$ se registró la preparación pre operatoria de la piel, de los cuales a la mayoría se les efectuó baño en cama como preparación (84,6\%). El 7,7\% de la cohorte presentó un foco infeccioso concomitante previo a la intervención quirúrgica, siendo la infección del tracto urinario la localización más frecuente, con 5,6\% del total de pacientes (Tabla 2).

Resulta importante destacar que los días de estada de los pacientes previos a la artroplastía fueron de casi 5

Tabla 1. Descripción general de pacientes intervenidos de prótesis total de cadera entre los años 2012 y 2014 en Complejo Asistencial Dr. Sótero del Río

\begin{tabular}{|c|c|c|c|c|c|c|}
\hline Descriptor & Min & P25 & Mediana & P75 & Máx. & RIC \\
\hline Edad (años) & 32 & 63 & 71 & 77 & 91 & 14 \\
\hline Mujeres & 32 & 63 & 71 & 78 & 91 & 15 \\
\hline Hombres & 32 & 63,5 & 70 & 76 & 89 & 12,5 \\
\hline Peso (kg) & 40 & 60 & 70 & 79 & 117 & 19 \\
\hline Mujeres & 40 & 58,5 & 66,5 & 74 & 109 & 15,5 \\
\hline Hombres & 50 & 69 & 76 & 85 & 117 & 16 \\
\hline Talla (cm) & 137 & 152 & 160 & 165 & 185 & 13 \\
\hline Mujeres & 138 & 150 & 155 & 160 & 177 & 10 \\
\hline Hombres & 137 & 163 & 168 & 172 & 185 & 9 \\
\hline IMC (kg/talla²) & 17,53 & 24,37 & 27,06 & 30,29 & 43,56 & 5,93 \\
\hline Mujeres & 17,53 & 24,35 & 27,41 & 30,86 & 43,56 & 6,51 \\
\hline Hombres & 17,72 & 24,59 & 26,21 & 29,34 & 40,48 & 4,75 \\
\hline
\end{tabular}

Tabla 2. Descripción factores asociados a la atención de salud preoperatorios de pacientes intervenidos de prótesis total de cadera entre 2012 y 2014 en Complejo Asistencial Dr. Sótero del Río

\begin{tabular}{|c|c|c|}
\hline Descriptor & $\mathbf{n}$ & $\%$ \\
\hline Hospitalización previa (si) & 47 & $10,66 \%$ \\
\hline Cirugía previa (si) & 151 & $34,24 \%$ \\
\hline \multicolumn{3}{|l|}{ Motivo prótesis } \\
\hline Artrosis & 337 & $76,42 \%$ \\
\hline Fractura & 98 & $22,22 \%$ \\
\hline Otros & 6 & $1,36 \%$ \\
\hline \multicolumn{3}{|l|}{ Servicio clínico pre cirugía } \\
\hline Traumatología & 353 & $80,05 \%$ \\
\hline Pensionado & 51 & $11,56 \%$ \\
\hline Otros & 37 & $8,39 \%$ \\
\hline Preparación preoperatoria & 278 & $72,02 \%$ \\
\hline Baño en cama & 143 & $84,62 \%$ \\
\hline Ducha & 26 & $15,38 \%$ \\
\hline Foco infeccioso concomitante & 34 & $7,71 \%$ \\
\hline Foco intra abdominal & 2 & $0,45 \%$ \\
\hline Infección urinaria & 25 & $5,68 \%$ \\
\hline Neumonía de la comunidad & 4 & $0,91 \%$ \\
\hline Neumonía intrahospitalaria & 1 & $0,23 \%$ \\
\hline Síndrome diarreico & 1 & $0,23 \%$ \\
\hline Tuberculosis nodular & 1 & $0,23 \%$ \\
\hline
\end{tabular}


días. En los pacientes intervenidos por artrosis, la mayoría presentó una estadía menor a 5 días, con un máximo de 20 días; en cambio, los pacientes intervenidos por fractura de cadera presentaron una mediana cercana a los 10 días, con un máximo de hasta 80 días. Esta diferencia fue estadísticamente significativa $(\mathrm{p}<0,001)$.

Solo en $63,2 \%$ de los pacientes intervenidos se cumplió la técnica de preparación intraoperatoria de la piel. Según lo descrito en registros clínicos, solo el 29\% de los pacientes presentaron un sangramiento anormal durante el acto quirúrgico. Respecto al tiempo operatorio, en general las cirugías duraron menos de 2,5 h, aunque la duración fue diferente respecto si el paciente presentaba artrosis o fractura, siendo mayor en los pacientes pertenecientes al último grupo. El tiempo de administración de la profilaxis antimicrobiana presentó una mediana de $30 \mathrm{~min}\left(\mathrm{P}_{25}=20\right.$; $\mathrm{P}_{75}=30 ; \mathrm{RIC}=10$ ). El $25 \%$ de los pacientes estudiados recibió antibioprofilaxis en un tiempo menor o igual a 20 min, al 80,5\% se les administró de forma correcta, es decir, alrededor de 20 a $30 \mathrm{~min}$ antes de la incisión quirúrgica. El antibacteriano más utilizado fue cefazolina, en $88,5 \%$ de los pacientes. El cumplimiento de la profilaxis según dosis por peso, refuerzo según tiempo operatorio, tipo de antimicrobiano administrado y tiempo de administración solo se cumplió en 29,7\% del total de pacientes. En los pacientes que presentaron una infección de herida quirúrgica el cumplimiento alcanzó solo $13,3 \%$.

\section{Análisis univariado por medio de regresión logística}

El modelo univariado se presenta en la Tabla 3.

\section{Análisis multivariado por medio de regresión logística y determinación de modelo predictivo}

Considerando el modelo univariado obtenido, se efectuó el análisis multivariado con las variables mencionadas con anterioridad y se efectuó el cruce nuevamente con variables de importancia según la literatura especializada. Se aplicaron los criterios de eliminación descritos en el método del estudio, obteniéndose el siguiente modelo (Tabla 4).

El modelo presentó un pseudo $\mathrm{R}^{2}=0,2293$, entendiéndose que explica alrededor de $23 \%$ de los casos de IHO en los sujetos incluidos para el análisis. Además, se manifiesta que el modelo predictivo presenta una adecuada bondad de ajuste para los sujetos incluidos en él ( $\chi^{2}$ de Pearson $=48,57$, junto a un $\mathrm{p}=0,6832$ ). Posteriormente al efectuar el cruce de la totalidad de las variables incluidas en el modelo, ninguna de ellas presentó el mismo efecto sobre la variable respuesta o dependiente (IHO), es decir,

Tabla 3. Análisis univariado de variables independientes por regresión logística para infección de herida operatoria en pacientes intervenidos de artroplastía total de caderas durante los años 2012 al 2014 en Complejo Asistencial Dr. Sótero del Río

\begin{tabular}{|c|c|c|c|c|}
\hline Variables Independientes & OR & Error estándar & P-value & IC $95 \%$ \\
\hline Diagnóstico de espondilodiscitis previo a cirugía & 23,39 & 33,55 & 0,028 & $1,41-389,14$ \\
\hline Diagnóstico de cardiopatía previo a cirugía & 24,71 & 25,45 & 0,002 & $3,28-186,06$ \\
\hline Diagnóstico de depresión previo a cirugía & 4,21 & 2,84 & 0,033 & $1,12-15,76$ \\
\hline Tratamiento antibiótico con imipenem previo a cirugía & 23,39 & 33,55 & 0,028 & $1,41-389,14$ \\
\hline Tratamiento antibiótico con amikacina previo a cirugía & 23,39 & 33,55 & 0,028 & $1,41-389,14$ \\
\hline Sangramiento anormal en intraoperatorio & 3,62 & 1,74 & 0,007 & $1,42-9,26$ \\
\hline Administración de glóbulos rojos en post operatorio & 4,01 & 1,92 & 0,004 & $1,57-10,26$ \\
\hline Estadía Unidad Paciente Crítico en post operatorio & 24,53 & 25,27 & 0,002 & $3,26-184,74$ \\
\hline
\end{tabular}

Tabla 4. Modelo predictivo por regresión logística multivariado para infección de herida operatoria en pacientes intervenidos de artroplastia total de caderas durante los años 2012 al 2014 en Complejo Asistencial Dr. Sótero del Río

\begin{tabular}{|c|c|c|c|c|c|}
\hline Variables Independientes & OR & Coeficiente (B) & Error estándar & P-value & IC $95 \%$ \\
\hline Días de estadía post operatoria & 1,11 & 0,103 & 0,04 & 0,004 & $1,03-1,20$ \\
\hline Transfusión de al menos una unidad de glóbulos rojos & 3,13 & 1,140 & 1,76 & 0,042 & $1,17-10,86$ \\
\hline Diagnóstico médico de depresión previo a intervención & 5,75 & 1,750 & 4,35 & 0,021 & $1,32-25,32$ \\
\hline Cumplimiento del tiempo de administración profilaxis antibiótica & 0,18 & $-1,698$ & 0,11 & 0,005 & $0,06-0,60$ \\
\hline
\end{tabular}


ningún par de variables testeadas presentó un efecto conjunto en la presencia de IHO en pacientes con ATC.

\section{Análisis del modelo predictivo por medio de curva ROC}

El modelo presentó una capacidad de discriminación de $80,7 \%$ (Figura 1).

\section{Análisis de sensibilidad de las pérdidas del modelo predictivo}

Según el análisis, los sujetos incluidos y no incluidos en modelo predictivo final son comparables, pero se debe especificar que dentro de los sujetos no incluidos existió mayor proporción de pacientes con hipertensión arterial. En el resto de variables comparadas no existen diferencias significativas (Tabla 5).

\section{"Score" de infección de herida operatoria en} pacientes intervenidos de prótesis total de caderas

Se obtuvo el siguiente "score" (Tabla 6).

Se observó que $55,6 \%$ de los pacientes infectados se encontraron entre 8 y 14 puntos.

La mediana del "score" en pacientes sin IHO se presentó alrededor de los 6 puntos, en cambio la mediana en pacientes con IHO se presentó alrededor de los 11-12 puntos. Es evidente que los pacientes con IHO presentaron un "score" más elevado en comparación con los sin IHO. (Figura 2).

Se estableció como punto de corte 13 puntos, con una sensibilidad de 44\%, especificidad de 91,6\%, LR (+) de 52.903 y LR(-) 0,6065 (Tabla 7).

Desde el punto de vista clínico, se estableció por consenso de los investigadores y opinión de médicos infectologos clínicos, la estratificación de los puntajes en los siguientes rangos: 1 a 6 puntos "bajo riesgo", 7 a 12 puntos "mediano riesgo", 13 a 18 puntos "alto riesgo", desde 19 puntos como "máximo riesgo".

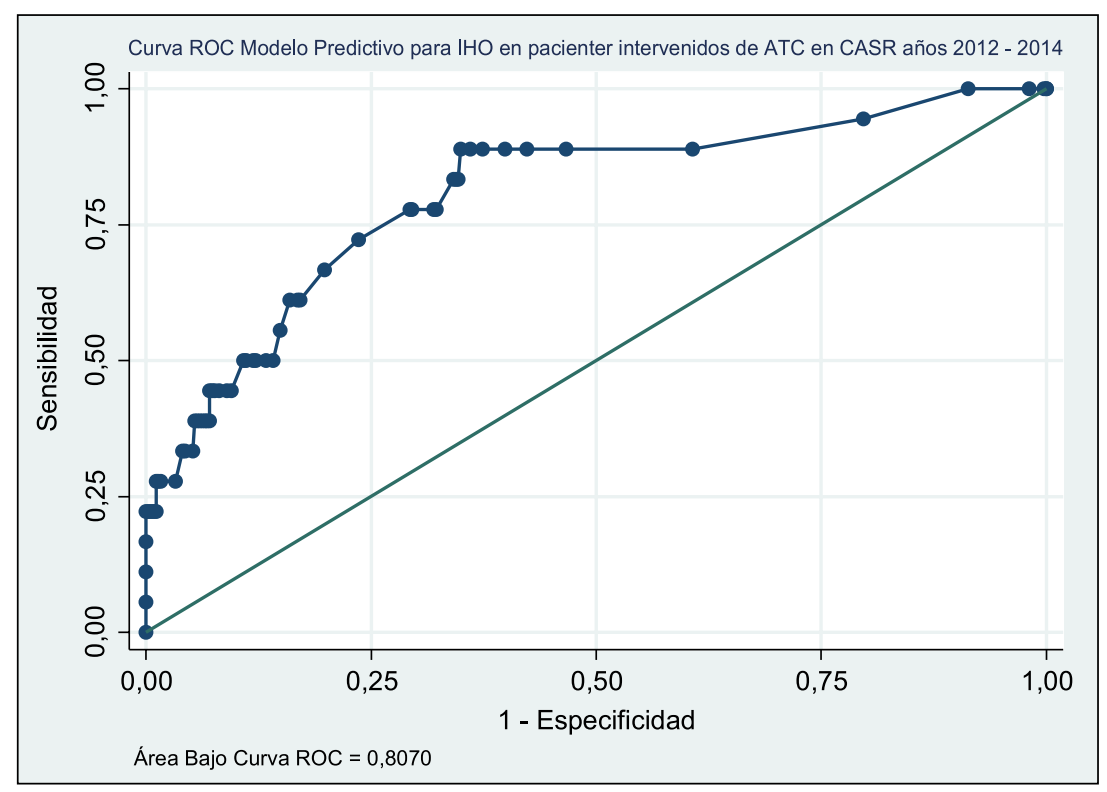

Figura 1. Curva ROC de modelo predictivo para infección de herida operatoria en pacientes intervenidos de prótesis total de caderas entre los años 2012 y 2014 en Hospital de Alta Complejidad.

\section{Discusión}

Antes de efectuar análisis del modelo predictivo, es necesario discutir respecto al número de días preoperatorios de pacientes que sufrieron una fractura. Esto se debe a que según la realidad de la institución en donde se efectuó el estudio, se requiere de estudios imagenológicos los que no siempre están disponibles de forma rápida, lo que aumenta la estadía pre-quirúrgica de ese tipo de pacientes.

Al observar el modelo predictivo obtenido, resalta que incluye variables que según la revisión de la literatura

\begin{tabular}{|c|c|c|c|}
\hline Descriptor & Sujetos incluidos en modelo & Sujetos no incluidos en modelo & P-value \\
\hline Media edad (años) & 68,90 & 70,61 & 0,325 \\
\hline Proporción mujeres & $68,73 \%(266)$ & $64,81 \%(35)$ & 0,562 \\
\hline Proporción sujetos con HTA & $58,91 \%(228)$ & $38,89 \%(21)$ & $0,005^{*}$ \\
\hline Proporción sujetos con diabetes mellitus & $17,57 \% \quad(68)$ & $12,96 \% \quad(7)$ & 0,398 \\
\hline Proporción motivo de prótesis por artrosis & $77,52 \%(300)$ & $68,52 \%(37)$ & 0,144 \\
\hline Media tiempo operatorio (horas) & 1,79 & 1,38 & 0,243 \\
\hline Porcentaje cumplimiento profilaxis antibiótica & $29,37 \%(111)$ & $60 \%(3)$ & 0,137 \\
\hline Porcentaje cumplimiento preparación intraoperatorioa & $64,34 \%(249)$ & $55,56 \%(30)$ & 0,210 \\
\hline
\end{tabular}




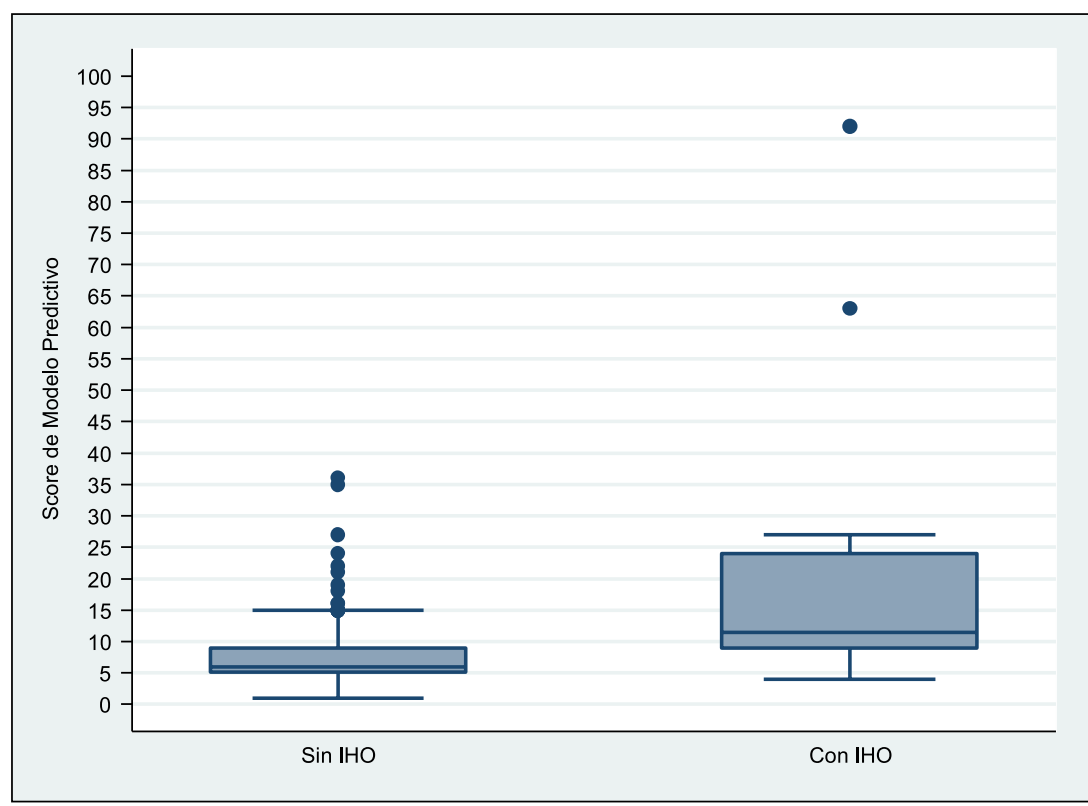

Figura 2. Score de infección de herida operatoria en pacientes intervenidos de artroplastía total de caderas en Hospital de Alta Complejidad años 2012 y 2014.

especializada, se presentan como factores asociados para una IHO en pacientes intervenidos de ATC $\mathrm{AT}^{2,4,10,16-18,20,22,26-30}$.

Respecto a incumplimiento en la antibioprofilaxis, concuerda con la literatura revisada que se presenta como un factor de riesgo ${ }^{12,23,27,31}$, pero en el presente estudio, solamente resulta significativo el incumplimiento del tiempo de administración, y no otros factores como el ajuste por peso o el refuerzo ante una cirugía prolongada o sangramiento excesivo. Este incumplimiento se debió principalmente a que los registros de la administración en algunas fichas clínicas no estaban presentes, o el tiempo fue menor a lo descrito a las recomendaciones vigentes durante el período de estudio (30 min previos a la incisión quirúrgica).

El riesgo que presenta el diagnóstico médico de depresión previo a la cirugía en los pacientes intervenidos también concuerda con la bibliografía revisada ${ }^{26}$. Según algunas publicaciones, la depresión puede causar déficit en el estado inmunológico del paciente, así como también, pérdida de interés en la ejecución de prácticas de la vida diaria, lo que puede influir en la higiene personal o en el seguimiento de las indicaciones post operatorias de una $\mathrm{ATC}^{26}$.

La administración de al menos una unidad de glóbulos rojos, se comportó como factor de riesgo en la cohorte en estudio. Según investigaciones revisadas, el sangramiento intraoperatorio y posterior anemia con hematocrito menor a $15 \%$ debieran ser un factor de riesgo ${ }^{17,31}$, pero en los registros clínicos revisados, esta variable estaba pobre-
Tabla 6. Descripción de "score" para infección de herida operatoria en pacientes intervenidos de artroplastia total de caderas en Complejo Asistencial Dr. Sótero del Río entre los años $2012-2014$

\begin{tabular}{|c|c|c|c|}
\hline "Score" & Sin IHO & Con IHO & Total \\
\hline 1 & 1 & 0 & 1 \\
\hline 2 & 6 & 0 & 6 \\
\hline 3 & 25 & 0 & 25 \\
\hline 4 & 43 & 1 & 44 \\
\hline 5 & 71 & 1 & 72 \\
\hline 6 & 59 & 1 & 60 \\
\hline 7 & 26 & 0 & 26 \\
\hline 8 & 30 & 1 & 31 \\
\hline 9 & 30 & 2 & 32 \\
\hline 10 & 23 & 1 & 25 \\
\hline 11 & 10 & 2 & 12 \\
\hline 12 & 14 & 1 & 15 \\
\hline 13 & 7 & 2 & 9 \\
\hline 14 & 8 & 1 & 9 \\
\hline 15 & 6 & 0 & 6 \\
\hline 16 & 2 & 0 & 2 \\
\hline 18 & 1 & 0 & 1 \\
\hline 19 & 1 & 0 & 1 \\
\hline 21 & 1 & 0 & 1 \\
\hline 22 & 1 & 0 & 1 \\
\hline 24 & 1 & 1 & 2 \\
\hline 25 & 0 & 1 & 1 \\
\hline 27 & 1 & 1 & 2 \\
\hline 35 & 1 & 0 & 1 \\
\hline 36 & 1 & 0 & 1 \\
\hline 63 & 0 & 1 & 1 \\
\hline 92 & 0 & 1 & 1 \\
\hline Total & 369 & 18 & 387 \\
\hline
\end{tabular}

mente descrita, por lo que quizás en algunos pacientes no se pudo obtener de forma correcta, dado que durante el intraoperatorio los registros de sangramiento son escasos y en el post-operatorio no se pudo determinar el valor del hematocrito en algunos pacientes, por no encontrarse dicha detección en los registros clínicos analizados. Es probable que la administración de glóbulos rojos en la cohorte estudiada se muestre como un "proxy" del sangramiento intraoperatorio y posterior anemia, dado que es dudoso que solo el hecho de recibir una transfusión genere algún mecanismo que pudiera aumentar el riesgo de una IHO, dado que el proceso de la preparación y administración 


\begin{tabular}{|c|c|c|c|c|c|}
\hline Punto de corte & Sensibilidad & Especificidad & Correctamente clasificados & LR+ & LR- \\
\hline$(>=1)$ & $100,00 \%$ & $0,00 \%$ & $4,65 \%$ & 10.000 & - \\
\hline$(>=2)$ & $100,00 \%$ & $0,27 \%$ & $4,91 \%$ & 10.027 & 0 \\
\hline$(>=3)$ & $100,00 \%$ & $1,90 \%$ & $6,46 \%$ & 10.193 & 0 \\
\hline$(>=4)$ & $100,00 \%$ & $8,67 \%$ & $12,92 \%$ & 10.950 & 0 \\
\hline$(>=5)$ & $94,44 \%$ & $20,33 \%$ & $23,77 \%$ & 11.854 & 0,2733 \\
\hline$(>=6)$ & $88,89 \%$ & $39,57 \%$ & $41,86 \%$ & 14.709 & 0,2808 \\
\hline$(>=7)$ & $83,33 \%$ & $55,56 \%$ & $56,85 \%$ & 18.750 & 0,3 \\
\hline$(>=8)$ & $83,33 \%$ & $62,60 \%$ & $63,57 \%$ & 22.283 & 0,2662 \\
\hline$(>=9)$ & $77,78 \%$ & $70,73 \%$ & $71,06 \%$ & 26.574 & 0,3142 \\
\hline$(>=10)$ & $66,67 \%$ & $78,86 \%$ & $78,29 \%$ & 31.538 & 0,4227 \\
\hline$(>=11)$ & $61,11 \%$ & $85,09 \%$ & $83,98 \%$ & 41.000 & 0,457 \\
\hline$(>=12)$ & $50,00 \%$ & $87,80 \%$ & $86,05 \%$ & 41.000 & 0,5694 \\
\hline$(>=13)$ & $44,44 \%$ & $91,60 \%$ & $89,41 \%$ & 52.903 & 0,6065 \\
\hline$(>=14)$ & $33,33 \%$ & $93,50 \%$ & $90,70 \%$ & 51.250 & 0,713 \\
\hline$(>=15)$ & $27,78 \%$ & $95,66 \%$ & $92,51 \%$ & 64.062 & 0,755 \\
\hline$(>=16)$ & $27,78 \%$ & $97,29 \%$ & $94,06 \%$ & 102.500 & 0,7423 \\
\hline$(>=18)$ & $27,78 \%$ & $97,83 \%$ & $94,57 \%$ & 128.125 & 0,7382 \\
\hline$(>=19)$ & $27,78 \%$ & $98,10 \%$ & $94,83 \%$ & 146.429 & 0,7362 \\
\hline$(>=21)$ & $27,78 \%$ & $98,37 \%$ & $95,09 \%$ & 170.834 & 0,7342 \\
\hline$(>=22)$ & $27,78 \%$ & $98,64 \%$ & $95,35 \%$ & 204.999 & 0,7321 \\
\hline$(>=24)$ & $27,78 \%$ & $98,92 \%$ & $95,61 \%$ & 256.249 & 0,7301 \\
\hline$(>=25)$ & $22,22 \%$ & $99,19 \%$ & $95,61 \%$ & 273.335 & 0,7842 \\
\hline$(>=27)$ & $16,67 \%$ & $99,19 \%$ & $95,35 \%$ & 205.001 & 0,8402 \\
\hline$(>=35)$ & $11,11 \%$ & $99,46 \%$ & $95,35 \%$ & 205.002 & 0,8937 \\
\hline$(>=36)$ & $11,11 \%$ & $99,73 \%$ & $95,61 \%$ & 409.998 & 0,8913 \\
\hline$(>=63)$ & $11,11 \%$ & $100,00 \%$ & $95,87 \%$ & - & 0,8889 \\
\hline$(>=92)$ & $5,56 \%$ & $100,00 \%$ & $95,61 \%$ & - & 0,9444 \\
\hline$(>92)$ & $0,00 \%$ & $100,00 \%$ & $95,35 \%$ & - & 10,000 \\
\hline
\end{tabular}

de transfusiones cuenta con altos estándares de calidad, junto a controles virológicos y bacteriológicos.

El último factor asociado determinado en el modelo predictivo fue el tiempo de estadía post-operatoria, concordante con lo ya descrito ${ }^{32}$, aunque en otro estudio, también se comporta como factor de riesgo los días totales de estadía hospitalaria ${ }^{10}$. El mayor tiempo post-operatorio, por lo general, puede significar que el paciente tuvo alguna complicación en su recuperación no pesquisada ni estudiada como variable al momento de la recolección de información, lo que realmente puede comportarse como factor de riesgo. Nuevamente quizás esta variable se com- porta como un "proxy" de los motivos de prolongación de la estadía hospitalaria post-operatoria no estudiadas en la presente investigación.

El modelo predictivo se ajusta bastante bien a la cohorte estudiada, comprobado con estadísticos diagnósticos de bondad de ajuste, así como por su sensibilidad y especificidad, expuesto en la gráfica o curva ROC, con un área bajo la curva mayor a 0,8 , por lo que demuestra que el modelo explica mucho mejor que el azar la ocurrencia de las IHO en los pacientes intervenidos. Creemos que el presente modelo será de gran contribución a la práctica clínica habitual, por lo que se podrá intervenir en los 
pacientes que presenten dichos factores de riesgo, con el fin de prevenir una IHO.

Respecto a la escala de riesgo o "score" determinado, se presenta como una importante herramienta en el ámbito de la prevención de las IHO y en la práctica clínica diaria, dado que se puede analizar de forma individual el riesgo de cada uno de los pacientes respecto a presentar uno de estos episodios.

Es importante destacar que, según los factores de riesgo descritos en el modelo predictivo, se puede intervenir directamente en el tiempo de administración de la antibioprofilaxis, así como en los días de estada postquirúrgicos, mejorando la gestión del alta y la articulación de la red asistencial. Sobre la administración de glóbulos rojos, se debe protocolizar dicha indicación, con el fin de efectuar transfusión a los pacientes que efectivamente lo requieren, aunque este punto es un factor de riesgo un poco más complejo de intervenir. Sobre el diagnóstico previo de depresión del paciente, es una co-morbilidad no modificable.

Las limitaciones principales del estudio es que fue basado únicamente en registros clínicos, el que solo se haya realizado en un centro hospitalario, por lo que hubo pérdida de información respecto a diversas variables, asî como no se puede asegurar que el simple hecho de que las actividades o situaciones no hayan sido registradas, efectivamente no hubieran sido efectuadas, así como también, el registro clínico en algunas oportunidades se efectúa de forma posterior a los hechos, incluyendo un sesgo de memoria en los resultados obtenidos

\section{Conclusiones}

El modelo predictivo obtenido incluyó las variables: días de estadía post-operatoria, transfusión de al menos una unidad de glóbulos rojos, diagnóstico médico de depresión previo a la intervención quirúrgica e incumplimiento del tiempo de administración de la antibioprofi- laxis. La capacidad de discriminación del modelo fue de $80,7 \%$. El punto de corte en "score" determinado fue de 13 puntos, logrando una sensibilidad de $44,4 \%$, especificidad de 91,6\%, LR (+) 5,29 y LR (-) 0,61, considerando además la siguiente clasificación: 1 a 6 puntos "bajo riesgo", 7 a 12 puntos "mediano riesgo", 13 a 18 puntos "alto riesgo", desde 19 puntos como "máximo riesgo".

\section{Resumen}

Introducción: Las infecciones de herida operatoria de pacientes intervenidos de artroplastía total de cadera, presentan una incidencia desde 2 a $5 \%$, generando impacto en la estadía hospitalaria, uso de recursos, antibioterapia prolongada y secuelas temporales o definitivas. Objetivo: Generar un modelo predictivo para la infección de herida operatoria en pacientes intervenidos de artroplastía total de cadera, entre los años 2012 y 2014, en un hospital de alta complejidad. Material y Método: Cohorte de pacientes con artroplastía total de cadera. Se efectuó la descripción de las variables epidemiológicas y se generó un modelo predictivo por regresión logística. Resultados: Se analizaron 441 pacientes. El modelo predictivo obtenido incluyó las variables: días de estadía post operatoria (OR 1,11 IC95\% [1,03-1,20]), transfusión de al menos una unidad de glóbulos rojos (OR 3,13 IC95\% $[1,17-10,86])$, diagnóstico de depresión previo a la cirugía (OR 5,75 IC95\% [1,32-25,32], incumplimiento del tiempo de administración de la antibioprofilaxis (OR 5,46 IC95\% $[1,68-17,78] ; \mathrm{p}<0,001)$ y pseudo $\mathrm{R}^{2}=0,2293$. Punto de corte de "score" de 13 puntos con sensibilidad 44,4\%, especificidad de 91,6\%, LR (+) 5,29, LR (-) 0,61, considerando además la siguiente clasificación: 1 a 6 puntos "bajo riesgo", 7 a 12 puntos "mediano riesgo", 13 a 18 puntos "alto riesgo", desde 19 puntos como "máximo riesgo". Conclusión: El modelo presenta una buena capacidad de predicción de infección de herida operatoria y representa adecuadamente a la cohorte en estudio.

\section{Referencias bibliográficas}

1.- Brenner P, Nercelles P, Pohlenz M, Otaíza F. Costo de las infecciones intrahospitalarias en hospitales chilenos de alta y mediana complejidad. Rev Chilena Infectol 2003; 20: 285-90.

2.- Londoño Á, Morales J, Murillas M. Características epidemiológicas y factores de riesgo relacionados con la infección en el sitio operatorio en procedimientos de cirugía general. Rev Chil Cirugía 2011; 63: 559-65.

3.- Iribarren B O, Álvarez C A, Rodríguez C C, Ferrada M M, Hernández V H, Dorn H L. Costo y desenlace de la infección de artroplastía de cadera: Estudio de caso y control. Rev Chilena Infectol 2007; 24: 125-30.

4.- Ercole F F, Chianca T C M. Infecção de sítio cirúrgico em pacientes submetidos a artroplastias de quadril. Rev Latino-Am Enfermagem 2002; 10: 157-65. (Fecha de acceso: 19 de diciembre de 2014). Disponible en: http://www.scielo.br/pdf/rlae/v10n2/10509. pdf

5.- Otaíza F, Orsini M, Pholenz M. Indicadores de referencia nacional de infecciones asociadas a la atención en salud (IAAS) vigentes desde Mayo de 2014. Santiago de Chile; 2014.
6.- Pérez O, Palanco L. Artroplastia total cementada en enfermedad degenerativa de cadera: Su evaluación clínica. Rev Cubana Ortop Traumatol 2008; 22: 1. (Fecha de acceso: 19 de diciembre de 2014). Disponible en: http://scielo.sld.cu/scielo.php?pid=S0864215X2008000100003\&script=sci_arttext

7.- Del Sel H, Lopreite F, Menutti M, Mana Pastrian D. Artroplastia total de cadera en secuelas de fracturas del acetábulo. Rev Asoc Argent Ortop Traumatol 2011; 76 (1): 14-19. (Fecha de acceso: 19 de diciembre de 2014). Disponible en: http://bases.bireme.br/cgibin/wxislind.exe/ iah/online/?IsisScript $=$ iah/iah.xis $\& s r c=$ google $\& b$ 
ase $=$ LILACS\&lang $=$ p\&nextAction $=1$ nk \&exprSe arch $=595390 \&$ indexSearch=ID.

8.- Kurtz S M, Lau E, Watson H, Schmier J K, Parvizi J. Economic burden of periprosthetic joint infection in the United States. J Arthroplasty 2012; 27(8 Suppl): 61-5.e1. doi: 10.1016/j.arth.2012.02.022.

9.- Ravi B, Croxford R, Hollands S, Paterson J M, Bogoch E, Kreder H, et al. Increased risk of complications following total joint arthroplasty in patients with rheumatoid arthritis. Arthritis Rheumatol 2014; 66: 254-63. doi: 10.1002/ art.38231.

10.- Urquhart D M, Hanna F S, Brennan S L, Wluka A E, Leder K, Cameron P A, et al. Incidence and risk factors for deep surgical site infection after primary total hip arthroplasty: a systematic review. J Arthroplasty 2010; 25: 1216-22.e3. doi: 10.1016/j.arth.2009.08.011.

11.- Lopreite F, de Bielke H S, Garabano G, Rodríguez G G, Oviedo A, del Sel H. Artroplastía total de cadera en pacientes con artritis reumatoide. Rev Asoc Argentina Ortop y Traumatol 2014; 79: 74-81. (Fecha de acceso: 19 de diciembre de 2014). Disponible en: http:// ojs.aaot.org.ar/ojsr/index.php/AAOTMAG/ article/view/166.

12.- Mangram A J, Horan T C, Pearson M L, Silver L C, Jarvis W R. Guideline for Prevention of Surgical Site Infection, 1999. Centers for Disease Control and Prevention (CDC) Hospital Infection Control Practices Advisory Committee. Am J Infect Control 1999; 27: $97-$ 132; quiz 133-4; discussion 96.

13.- Bowler P G, Duerden B I, Armstrong D G. Wound microbiology and associated approaches to wound management. Clin Microbiol Rev 2001; 14: 244-69.

14.- Otaíza F. Sistema de Vigilancia de las Infecciones Intrahospitalarias. Santiago de Chile: Ministerio de Salud; 1998. p. 49.

15.- Otaíza F, Orsini M, Pholenz M. Instrucciones sobre vigilancia de IAAS a contar de enero 2013. Santiago de Chile; 2013.

16.- Dale H, Skråmm I, Løwer H L, Eriksen $\mathrm{H}$ M, Espehaug B, Furnes O, et al. Infection after primary hip arthroplasty: a comparison of 3 Norwegian health registers. Acta Orthop 2011; 82: 646-54. DOI: $10.3109 / 17453674.2011 .636671$.
17.- Cordero-Ampuero J, De Dios M. What are the risk factors for infection in hemiarthroplasties and total hip arthroplasties? Clin Orthop Relat Res 2010; 468: 3268-77. doi: 10.1007/s11999010-1411-8.

18.- Sousa R, Muñoz-Mahamud E, Quayle J, Da Costa L D, Casals C, Scott P, et al. Is asymptomatic bacteriuria a risk factor for prosthetic joint infection? Clin Infect Dis 2014; 59: 41-7. doi: 10.1093/cid/ciu235.

19.- Berbari E F, Osmon D R, Carr A, Hanssen A D, Baddour L M, Greene D, et al. Dental procedures as risk factors for prosthetic hip or knee infection: A Hospital-Based Prospective Case-Control Study. Clin Infect Dis 2010; 50: 8-16.

20.- Bozic K J, Lau E, Kurtz S, Ong K, Rubash H, Vail T P, et al. Patient-related risk factors for periprosthetic joint infection and postoperative mortality following total hip arthroplasty in Medicare patients. J Bone Joint Surg Am 2012; 94: 794-800. doi: 10.2106/JBJS.K.00072.

21.- Yomayusa N, Gaitán H, Suárez I, Ibáñez M, Hernández P, Álvarez C, et al. Validación de índices pronósticos e infección del sitio quirúrgico en hospitales de Colombia. Rev Salud Pública (Bogotá) 2008; 10: 744-55.

22.- Paryavi E, Stall A, Gupta R, Scharfstein D O, Castillo R C, Zadnik M, et al. Predictive model for surgical site infection risk after surgery for high-energy lower-extremity fractures: development of the risk of infection in orthopedic trauma surgery score. J Trauma Acute Care Surg 2013; 74: 1521-7. doi: 10.1097/TA.0b013e318292158d.

23.- Bestard M P, Fernández Z R, Ramírez J M R, Alba I D. Factores predictivos de las infecciones posoperatorias. Rev Cubana Cir 2011; 50(3): 257-65. (Fecha de acceso: 16 de diciembre de 2014). Disponible en: http://scielo.sld.cu/scielo. php?script=sci_abstract\&pid=S0034$74932011000300002 \& \operatorname{lng}=$ es.

24.- Iglesias Cabo T, Escabias Macucha M, Aguilera del Pino A M. Universidad de Granada. Métodos de Bondad de Ajuste en Regresión Logística. Máster Oficial en Estadística Aplicada. Tabajo Fin de Máster. Curso académico 2012/2013. (Fecha de acceso:
16 de diciembre de 2014). Disponible en : http://masteres.ugr.es/moea/pages/tfm-1213/ tfm_iglesiascabo_tania/!

25.- Tudela P, Lacoma A, Prat C, Mòdol J M, Giménez M, Barallat J, et al. Predicción de bacteriemia en los pacientes con sospecha de infección en urgencias. Med Clin (Barc) 2010; 135: 685-90. doi: 10.1016/j. medcli.2010.04.009.

26.- Bozic K J, Ward D T, Lau E C, Chan V, Wetters N G, Naziri Q, et al. Risk factors for periprosthetic joint infection following primary total hip arthroplasty: a case control study. J Arthroplasty 2014; 29: 154-6. doi: 10.1016/j. arth.2013.04.015.

27.- Centers for Disease Control and Prevention. Surgical Site Infection (SSI) Event. January 2015; 9-27. Disponible en: https://apic.org/ Resource_/TinyMceFileManager/Academy/ ASC_101_resources/Surveillance_NHSN/ NHSN_9pscSSIcurrent_jan2015.pdf.

28.- Martin J A, Buckwalter J A. The role of chondrocyte senescence in the pathogenesis of osteoarthritis and in limiting cartilage repair. $\mathrm{J}$ Bone Joint Surg Am 2003; 85-A Suppl 2: 10610.

29.- Ravi B, Croxford R, Hollands S, Paterson J M, Bogoch E, Kreder H, et al. Increased risk of complications following total joint arthroplasty in patients with rheumatoid arthritis. Arthritis Rheumatol 2014; 66: 254-63. doi: 10.1002/ art.38231.

30.- Wu C, Qu X, Liu F, Li H, Mao Y, Zhu Z. Risk factors for periprosthetic joint infection after total hip arthroplasty and total knee arthroplasty in Chinese patients. PLoS One 2014; 9: e95300. doi: 10.1371/journal.pone.0095300.

31.- Albuhairan B, Hind D, Hutchinson A. Antibiotic prophylaxis for wound infections in total joint arthroplasty: a systematic review. J Bone Joint Surg Br 2008; 90: 915-9. doi: 10.1302/0301-620X.90B7.20498.

32.- Ercole F F, Machado Chianca T C, Duarte D, Ferreira Starling C E, Carneiro M. Infecção de sítio cirúrgico em pacientes submetidos a cirurgias ortopédicas: o índice de risco NNIS e predição de risco. Rev Latino-Am Enfermagem 2011; 19: 269-276. (Fecha de acceso: 15 de noviembre de 2014). Disponible: http://www. scielo.br/pdf/rlae/v19n2/pt_07.pdf 